\title{
Doctors' stories, patients' stories: a narrative approach to teaching medical ethics
}

\author{
Barbara Nicholas and Grant Gillett Bioethics Research Centre, Otago University, Dunedin, New Zealand
}

\section{Abstract}

Many senior doctors have had little in the way of formal ethics training, but express considerable interest in extending their education in this area. This paper is the report of an initiative in continuing medical education in which doctors were introduced to narrative ethics. We review the theoretical basis of narrative ethics, and the structure of and response to the two-day workshop.

\section{Introduction}

The development of ethics education within medical training is of relatively recent origin. There are a large number of practising doctors whose ethics education has been restricted to a few brief lectures on medical etiquette, and the powerful process of apprenticeship training in clinical practice. Yet, as medical practice changes in response to advances in technology, pressures from the community for a different quality of doctor-patient relationship, and political changes in health provision, doctors' interest in the ethical aspects of their practice continues to grow.

In response to the increasing demand from doctors for opportunities to work more in medical ethics, staff at the Bioethics Research Centre at Otago University developed a two-day workshop for doctors that was offered as part of the Glaxo Foundation for Medical Education series during 1995. We decided to move away from the more usual approach to medical ethics, with its emphasis on the principles of bioethics, and focus instead on a narrative approach to ethics. We chose this approach for several reasons. It is an approach which is respectful of the experience that participants would bring with them; participants' responses and contexts can be fully part of the conversation and exploration; traditional approaches from philosophy and the principles of bioethics are not excluded but become tools that may be used to explore the complexity of the situation; and in addition, issues of

\section{Key words}

Narrative ethics; continuing medical education; medical discourse. power and "voice", raised within the post-modern context in which we now work, can be integrated into the discussion. These are particularly relevant to medical ethics.

\section{The course}

AIMS

We had a number of educational aims in mind as we planned this course. Firstly we wanted to introduce participants to narrative ethics and a narrative understanding of medical knowledge and to explore with them the concepts of interpretation and discourse. Secondly we wanted to develop doctors' skills, in particular their ability i) to recognise the narrative nature of the medical encounter and their own interpretive framework; ii) to reflect critically upon those frameworks; and iii) to discover something of the gift received in listening to the narratives of others, within the interpretive framework of that other.

OUR THEORETICAL FRAMEWORK: NARRATIVE ETHICS Medical ethics has been dominated by the four principles approach. There is little doubt that this has provided us with some useful tools for analysis of situations, and a common language that names important issues. Its wide level of acceptance demonstrates its usefulness and accessibility. However, the limitations of principles are now becoming obvious. There are philosophical difficulties with criteria for application of the principles, problems with how one resolves conflict between principles, and an unease amongst practitioners arising from the realisation that the realities and practicalities of clinical practice are not paid sufficient attention. ${ }^{1}$ Health professionals point out that details of context and the specifics of relationship are an intrinsic part of ethical health care, yet are invisible within a method which seeks some sort of universal approach. These points of detail often add a richness to a medical situation which helps to clarify the ethical issues. For example: Aiden is a young man of seventeen with mild diabetes. He has coped very well with diet and exercise and oral treatment but is now found to have poorly controlled blood sugar levels. 
He refuses to go on insulin therapy despite the fact that his doctor tells him about the risks to his eyesight, blood system and life itself if his insulin levels remain uncontrolled.

Here we could start an ethical analysis in terms of autonomy and the duty to protect from harm, but that would be premature. To begin to appreciate the issues we need to fill in the gaps in this story. Why does Aiden not want treatment? What is his image of himself? How does he fit his diabetes into his life story? With whom does he identify his doctor - with his parents, with society as a whole, or does he see him as his own adviser? A resolution of the problem cannot be attempted without these narrative insights.

\section{Alternatives to principles}

A number of alternatives to principles have been proposed. A revisitation of casuistry has been suggested, most notably by Jonsen and Toulmin ${ }^{2}$ and an ethics of care proposed both from nursing ${ }^{3}$ and from feminist theory. ${ }^{4}$ At least one doctor has suggested that we need to turn to microethics. ${ }^{5}$ Casuistry recognises the central place given to story in the discernment of what is ethical in a particular context, but fails to pay attention to the frameworks that structure interpretation. The story in question is compared and contrasted at certain points with others and a comparable decision made about the moral issues. For those within a particular worldview or culture the method of comparing stories, and moving from the clear case to the more ambiguous one, may be a useful approach. However, as Kopleman has pointed out, ${ }^{6}$ casuistry does, in practice, give some judgments epistemic privilege, without specifying the criteria for those choices. The consequence is that casuistry can function to maintain the privilege of certain frameworks and accompanying assumptions, but does not provide any necessary critique of these.

An ethics of care attempts to do two main things: (i) to acknowledge the moral significance of the interpersonal relationship that develops between people, particularly when one person is vulnerable because of sickness or age, and (ii) to value as morally significant those virtues associated with care (such as empathy and compassion), and the physical expressions of care that have traditionally been carried out by women in their roles as mothers or nurses, but not recognised as moral work. The relevant virtues are seen as emerging, in part, from the experience of caring, not just from abstract thought. (This is also a central claim in Aristotelian moral theory.)

Microethics also focuses on particular situations and the details of the clinical transaction, and therefore uses features of narrative to guide judgment.

Narrative ethics has emerged in conjunction with these approaches, recognising (as does casuistry) the centrality of story or narrative in ethics, and grappling with the specifics of context and relation $\overrightarrow{\bar{z}}$. ship (also a focus of the ethic of care). In additions? narrative ethics recognises that questions of interpretation and power are a necessary part of ethics.

An important contributor in this area is Kathryn Hunter, who in her book Doctors' Stories ${ }^{7}$ identifies the narrative nature of medical knowledge. She illuminates the place of story in medicine, and thes extent to which it structures medical knowledg 5 and plays a central role in the transmission of thisknowledge. Story is integral to many parts of medicatw life. Story forms the basis of medical care in the narratives that patients bring to their doctors and in the narrative the doctor constructs in relation to the्w patient. Story also structures the conversations between health professionals and provides a majote vehicle for the transmission of knowledge and the formation of a professional.

A number of interpretive frameworks may be operating at the same time. The doctor will be

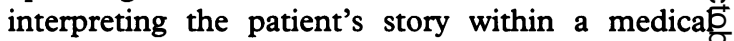
framework, and be able to offer a new narrative back to the patient that may help the patient make sense or meaning of what is happening to him or her. Ate the same time, a patient will need to find a way todit the narrative offered by the doctor, and their experito ence of care, into their total life narrative. Howeverts the patient's life narrative includes many things tha are beyond the reach of the health professional's gaze. Patients are part of a network of relationships and responsibilities. Particular choices, say about $\overrightarrow{\vec{b}}$ what course of treatment to seek, are not made in 3 isolation from other choices and responsibilities in peoples' lives but in conversation with them. Hunter. argues: "Because narrative depicts events embeddeकs in the lives and ongoing concerns of the protago $\$$ nists, it represents moral choice not as a snapshos but as an infolding web of character and motivation. luck and circumstance - with all the apparent nonessentials that give human beings their sense of identity. In its representation of subjective experience, narrative gives us access to the perceptions an $b$ valuation of other human beings, and thus narrative. bioethics is a means of thinking about the meanings of illness in the life of a patient and about the role of the physician in the patient-physician interaction".

\section{Total narrative}

A narrative ethic recognises that events (such as? decisions about whether or not to consent to chemotherapy) are embedded in the total narrative of people's lives. Clinical choices are not isolated from all else that happens in people's lives but are्ष part of an ongoing narrative. Moral choices made $\frac{O}{2}$ like all decisions, are part of an effort to live a life that has coherence and meaning. The health professional and the patient do not make choices on 
the basis of abstract principles (although these may aid reflection) but on the basis of their efforts to live a life with some sort of integrity.

This approach to ethics recognises that human understanding is socially situated, that we develop our sense of what is right or appropriate, what is good or to be resisted, out of our total life experience - the people we have known, the previous experiences we have had, the ideas and frameworks that have made sense in the past. Rather than trying to find an approach which escapes from or removes the specifics of culture, history or relationship, a narrative ethic seeks to make visible these particulars, and to take seriously the messy and complicated business of negotiating meaning and action. The meaning of an event, and the action to be taken, must embody the framework of goodness which that person inhabits.

This is highly dependent on the discourses which have provided the person concerned with valueladen terms and significations (ways of describing or naming which express significance) applicable to her or his life experience. For instance, consider an older man, weakened by cancer and unable to make any "contribution" to his family's life in terms of physical work. Terms that he might use are "good for nothing", "layabout", "just sitting on my backside all day", and so on. Offered a chance to do something about this - to end his life, say - he might respond to that choice by using these very actionorientated significations to guide his decision.

\section{Medical humanities}

This re-affirmation of medicine as more than scientific knowledge and technical skill is also there in the contemporary moves towards more training in medical humanities. However, what makes narrative ethics more than medical humanities in a fresh dress is its recognition that issues of character and intent, while important, are not everything. As a professional a doctor needs also to address issues of power and discursive context as they relate to interpretation and construction of medical narrative. A narrative ethic recognises that we make choices of value and moral judgment when we privilege one narrative or interpretive framework over another. A narrative ethic asks: whose story is being told, and by whom? whose interpretive framework is being given authority? and how do those of us with social or institutional power respond to narratives with which we are uncomfortable or which challenge our position??

A narrative ethic may also recognise that the "voice" of those on the margins of a society or discourse, in their narratives and interpretations, has something to offer those, such as doctors, in a more central position, and may alert us to aspects of practice which are experienced in ways that are not intended. Doctors, however well-intentioned and user-friendly, are foci of social, personal and expert power, and must therefore be aware of that fact and its influence on the clinical encounter. This aspect of a narrative ethic is perhaps one of the more radical features of this approach and one that has been explored by several feminist writers. ${ }^{10}$ It assumes that ethics is not just about regulating the worst excesses of a social practice such as medicine, or even humanising the practice within the boundaries already functioning. Narrative ethics is also concerned with transformation, with reflecting upon the fundamental assumptions of a practice or discourse, the impact of those assumptions upon the most vulnerable, and with transforming the practice to incorporate the insights and interpretive priorities of the marginalised.

\section{Method}

In planning the workshop on narrative ethics, it was clear to us that our method needed to focus on narrative, and to incorporate largely experiential learning processes. There was some theoretical input, but it was kept to a minimum. In the first session we asked participants to talk in groups of two or three about a clinical interaction they felt good about and then in wider discussion we identified some of the themes that emerged when doctors discussed work that had gone well. The concepts of medical discourse, power and position were briefly introduced, drawing on continental philosophy and discourse analysis, in terms used by thinkers such as Michel Foucault. Then we again asked the participants to tell stories. This time we asked them to tell about the most interesting patient story they had heard. Kathryn Hunter's work about the narrative nature of medical knowledge was then introduced, and the variety of narratives that are told concerning the patient. These include the patient's story, the notes, the test results, and the charts. And these are all interpreted in the light of previous stories within medicine - the anecdotes, the scientific literature, stories of previous patients that seem similar. The group moved on to identify some of the sources of information that they used to construct their stories. They began to identify the criteria that they were using in deciding what to omit, and what to include.

In the second session, the participants were divided into three groups, and each given a different set of case notes (fictional, but a composite of "real" cases). Each of those groups then divided in half. Their task was to decipher the doctor's handwriting, and prepare a creative presentation of the case. One half were to present it as a story told to other doctors, by doctors. The other half were to present the story as told by the patient to friends or family members.

In the third session these cases were presented. The results were wonderful - both entertaining and instructive. For instance, one of the cases concerned a young unmarried mother of three, referred because 
of an offensive vaginal discharge. The notes recorded that the patient was uncommunicative, and would not provide the name of her sexual partner. Swabs were taken to test for clamydia, but she left her prescription behind. On a subsequent visit a week later, the notes recorded her as sullen and resentful, uncooperative with questioning, still not cooperative with contact tracing, possibly a noncompliant patient. The "narratives" told by the two groups spoke of two different worlds, two totally different interpretive frameworks, two different sets of priorities and concerns. It became clear that the woman was not able to receive good care because the doctor was not able adequately to imagine the world she inhabited and the choices with which she was dealing. The doctor was concerned with compliance. The woman was concerned with survival of herself and her children.

On the second day we turned from the medical narratives to narratives found in the literature, but relating to medicine. Three extracts or stories were chosen. The material had been sent out to participants prior to the course, and all had had an opportunity to re-read it overnight at the conclusion of the previous sessions. Each extract was discussed in groups of five or six, and then reflections brought back to the whole group for wider discussion. Each group was asked to select a reporter to present the group's reflections back to the larger group.

\section{Parallels}

The first narrative was from the corpus of medical humanity literature, William Carlos Williams's "The use of force", from his collection The Doctor Stories. ${ }^{11}$ Doctors present had no difficulty identifying with the doctor in the story, and in recognising his dilemmas, choices and feelings. The narrative was one with which they could easily make connections, and recognise parallels in their own practice. Participants then also engaged with the experience of the patient and her parents. They made real efforts to construct the narrative as it might be experienced or told by these other people in the story.

The second narrative presented more challenges. We used a story by Sarah Maitland, "Forceps delivery", in her collection of short stories Women Fly When Men Aren't Watching. ${ }^{12}$ This is a fictionalised re-telling of a tale from medical history, that of the English doctor Dr Hugh Chamberlain's demonstration of the forceps to a French doctor, Dr Francois Mariceau, in an effort to sell this, until then, secret device. But this narrative is not in the voice of one of the doctors involved, but through the voice of the woman dwarf on whom the forceps were demonstrated. It is the patient's experience that comes first. This was a difficult story for some of the doctors on the course. It seemed more fantastic, more outrageous than the previous tale. It was more of a challenge to identify with the person telling the story. Nor were they sure they wanted to identify with the doctors of whom she was speaking.

This narrative is part of medical history, a story: already told in some obstetric textbooks. But as told there the woman is nameless, almost invisible? lacking history or connection. Hearing the story in the woman's voice emphasised to the participants? the impact on the narrative of the position of the teller, and the way in which aspects of patienty experience are "written out" or silenced in dominantnarratives of medicine. As they struggled with this narrative, participants engaged with issues of consent, silence and compliance in new ways, and began to identify parallel situations in contemporary practice.

The third narrative we used was another told by $a_{U}^{\omega}$ doctor. This was an extract from A J Cronin's The Citadel ${ }^{13}$; the story of the botched operation, the death of a patient, and the operating and referring doctors' different responses. None of those present saw this story as at all fantastic or exaggerated.O All appeared to identify readily with the type of situation, and the difficulties of responding to कD colleague's unacceptable practice. Some had read the full novel, for others this was a first encountere with the text. There was, in the discussion of $N$ this third story, a willingness to imagine what narrative might be as told by the patient's familyos greater ease with re-framing the narrative, noticings what difference it might make to tell it from out of the experience of different participants.

Using the three narratives evoked discussion and exploration of a number of ethical issues. Each of the stories raised particular issues around medical practice; consent and treatment of children and relationships with their parents; doctor-patiens relationships; consent to innovative treatments compliance; honesty with patients; professiona responsibility and the impaired doctor. All these are regular topics in medical ethics, and the use of literature presented these issues in fresh ways to the participants. But additional ethical issues were raised. Whose voice were we hearing and payings attention to? Through what framework were we. filtering narratives? And on what basis did wêু exclude or include information and judge its signifi cance? As the workshop progressed participant revealed increased willingness to take seriously andw respect the differences that are present between the dominant narratives, and those that are told by the patient. They began to recognise that the doctor's power to tell a medical narrative can not only help to provide meaning and hope for those who are ill, but can also act to silence and ignore features of क्ष patient's life that are ethically significant to the patient, and should also be to the doctor. Not only new sensitivities are required to attend to this, but more self-conscious and self-critical understanding and use of medical narrative and power. 


\section{Discussion}

As presenters we put a lot of effort into creating an environment where participants could tell their own stories and listen to those of others, give and receive these stories as gift and experience an opportunity to reflect in a critical and constructive way upon their own interpretive frameworks, their limitations and their strengths. As educators we were aware that our educational method needed to be consistent with the theoretical method we were presenting. The medium was the message. We could not advocate the importance of narrative and of listening to those narratives that are different from our own if we did not offer a context within which participants' own narratives could be valued.

During the workshop the authors found it difficult to assess how participants were experiencing the process. Body language seemed incredibly controlled, but all contributed fully and offered no resistance to any of the activities. However, the anonymous evaluations at the end of the course were extremely positive. Participants found the course "practical, thought- provoking and understandable". One participant reported that narrative ethics re-affirmed the approach they had been using but provided some theoretical frameworks within which to understand and reflect upon ethical aspects of practice.

Narrative ethics does not replace other approaches to bioethics. The language of the principles or of philosophy can provide some important tools of analysis, casuistry may well reflect how a diverse group of people can develop consensus, and the concerns of an ethic of care highlight aspects of personal relationships that can easily be lost in a search for overarching theories or principles. However, a narrative ethic adds to our discussions of the ethics of health care. It creates an opportunity for critical reflection upon our theoretical frameworks and working assumptions that is grounded in the realities of peoples' lives. Not only philosophical integrity or tidiness is at stake in bioethics. We are talking about actual lives, real people. Narrative ethics reminds us of this and alerts us to how our institutional and professional practices are experienced by both health care provider and by patient or consumer. However, these individual experiences are only part of the conversation. Narrative ethics also invites us to place these experiences within the wider discourse of medicine, to reflect upon the social, political and historical context within which we live, and to make active, and ethical, choices about our own role in maintaining or transforming those discourses.

\section{Acknowledgement}

The workshop reported in this paper was sponsored by Glaxo Foundation for Medical Education.

Barbara Nicholas, $P h D$, is Lecturer at the Bioethics Research Centre, Otago University, Dunedin, New Zealand. Grant Gillett, $M B C h B$, FRACS, D Phil, is Professor in Medical Ethics at the Bioethics Research Centre, Otago University, Dunedin, New Zealand.

\section{References and notes}

1 The literature debating these issues is wide and extensive. See, for example, Clouser D, Gert B. A critique of principlism. Fournal of Medicine and Philosophy 1990; 15,2: 219-36; Green R. Method in bioethics: a troubled assessment. Fournal of Medicine and Philosophy 1990; 15,2: 179-97; Beauchamp TL, Childress JF. Principles of biomedical ethics [4th ed]. Oxford: Oxford University Press, 1994; and Gillon R, Lloyd A. Principles of health care ethics. Chichester: John Wiley and Sons, 1994.

2 Jonsen A, Toulmin S. The abuse of casuistry. Berkeley, Los Angles, London: University of California Press, 1988.

3 For example, Fry ST. Towards a theory of nursing ethics. Advances in Nursing Science 1989; 11,4: 9-22; Cooper M C. Principle-orientated ethics and the ethic of care: a creative tension. Advanced Nursing Science 1991 ; 14,2: 22-31.

4 See, for example, Larrabee MJ. An ethic of care. New York: Routledge, 1993, and Tronto J. Moral boundaries. New York, London: Routledge, 1993.

5 Komesaroff P. Troubled bodies. Melbourne: Melbourne University Press, 1996.

6 Kopelman LM. What is applied about applied philosophy? Fournal of Medicine and Philosophy 1990; 15: 199-218.

7 Hunter K. Doctor's stories. Princeton, New Jersey: Princeton University Press, 1991.

8 See reference 7: 101 .

9 Frank A. Editor's introduction: case histories and the ethics of voice. Second Opinion 1994; 17,3: 44-8.

10 Cannon K. Black womanist ethics. Atlanta: Scholars Press, 1988; Sherwin S. No longer patient. Philadelphia: Temple University Press, 1992; Welch S. A feminist ethic of risk. Minneapolis: Fortress Press, 1990.

11 Williams WC. The doctor stories. London, Boston: Faber and Faber, 1984.

12 Maitland S. Women fly when men aren't watching. London: Virago, 1993.

13 Cronin AJ. The citadel. London: New English Library, 1983. 